\title{
Consideraciones sobre la dispersión de la broca del café Hypothenemus hampei en relación con el empleo de trampas con atrayente. Una revisión
}

\section{Considerations about dispersion of the coffee berry borer Hypothenemus hampei related to the use of attractan-baited traps. A review}

DANIEL RODRÍGUEZ1,2

JOSÉ R. CURE H. ${ }^{1}$

FERNANDO GANTOR R. ${ }^{1}$

Fruto de café brocado.

Foto: D. Rodríguez

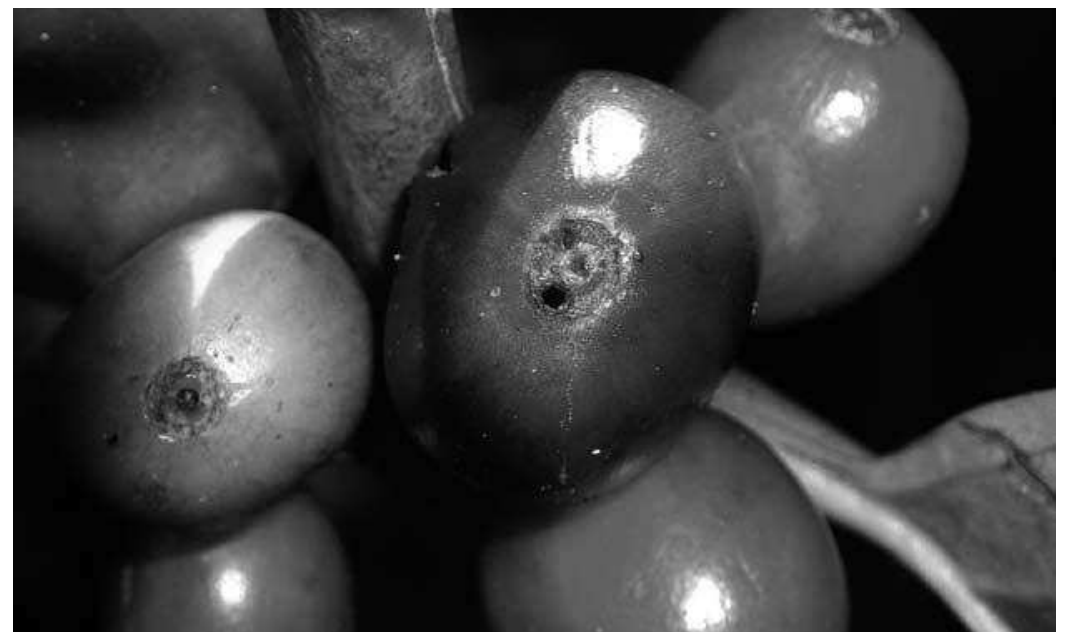

\section{Resumen}

En este trabajo se presenta una revisión acerca de la investigación realizada en torno a los aspectos biológicos de la migración y establecimiento de la broca del café, como un aspecto relevante para el desarrollo de métodos de captura en trampas, útiles para fines de monitoreo o control de la plaga. La discusión en relación con la broca del café es complementada con información disponible para otros Scolytinae de zonas con estaciones, que presentan características de comportamiento similiares. A partir del diagnóstico realizado se proponen temas de investigación relevantes para próximos trabajos en esta temática.

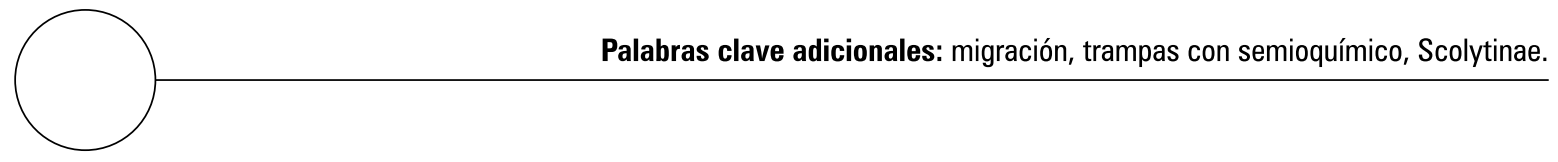

\footnotetext{
Facultad de Ciencias, Universidad Militar Nueva Granada, Bogotá (Colombia).

2 Autor para correspondencia.ecologia@umng.edu.co
} 


\section{Abstract}

A review about the research carried out on the biological topics of migration and establishment of the coffee berry borer is presented in this work, as an important topic to know in order to develop catch methods based on the use of traps, which can be useful for monitoring or control of the pest. The discussion about the coffee berry borer is complemented with information of Scolytinae from zones with seasonal climate, which presents a similar behavior. According to the diagnostics obtained, some research topics are proposed for future studies.

Additional key words: migration, traps lured with semiochemical, Scolytinae.

Fecha de recepción: 14-08-2008

Aprobado para publicación: 02-12-2008
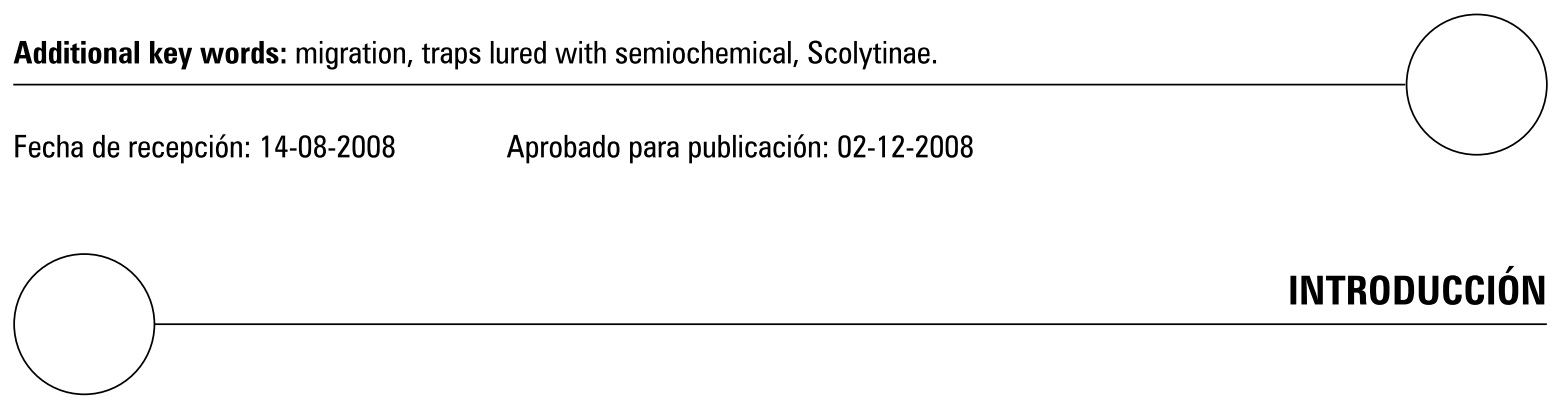

INTRODUCCIÓN

Una estrategia para el control de la broca del café que ha venido implementándose con resultados variables, es el empleo de trampas cebadas con una mezcla de alcoholes como atrayente. La idea de emplear trampas para la captura de broca nace del uso de esta estrategia para el manejo de otros Scolytinae de cultivos forestales (Moeck, 1970; Moeck, 1981; Lindgren, 1983), con base en principios de comportamiento similares. Desde entonces se han realizado estudios de comportamiento para identificar los estímulos que determinan la atracción de la broca hacia los frutos (Ticheler, 1963; Prates, 1969; Mendoza, 1991; Mathieu et al., 2001; Ortíz et al., 2004), y otros estudios con el propósito de definir las condiciones óptimas para el uso de la estrategia en campo (Mendoza, 1991; Mathieu, 1997b; González y Dufour, 2000; Borbón et al., 2000; Cárdenas, 2000; Villacorta et al., 2001; Barrera et al., 2004; Barrera et al., 2005; Fernández y Cordero, 2005; Cardona y Bustillo, 2006; Cardona y Bustillo, 2007; Dufour y Frérot, 2008), considerando factores como el atrayente empleado, la tasa de liberación del mismo, el color de la trampa, su diseño, la altura a la cual deben colocarse las trampas y el espaciamiento de estas en el cultivo. Sin embargo, existen discrepancias importantes entre los hallazgos de diferentes autores en cuanto a las características óptimas del muestreo en campo, y en cuanto a la eficiencia de las trampas como estrategia de control. Un aspecto que resulta de gran relevancia para obtener una mejor comprensión de la interacción entre la broca y las trampas con atrayente, es el conocimiento de los aspectos biológicos asociados a la migración y detección de nuevos hospederos, tema que se desarrolla en la presente revisión. El empleo de las trampas con atrayente se basa en utilizar los mecanismos propios de la plaga para realizar el proceso de selección del hospedero. Esta selección puede ser vista como parte de un proceso más amplio, que en el contexto de los Scolytinae plaga, de especies forestales, ha sido definido por Wood (1982), como la estrategia de colonización del hospedero. Debido a las importantes similitudes en algunos aspectos de la biología de estos insectos plaga de los forestales y la broca, en la presente revisión se ha empleado el modelo propuesto por este autor como herramienta de aproximación al caso de la broca. La estrategia de colonización consta de las etapas que se describen detalladamente a continuación.

\section{DISPERSIÓN}

Inicia con el abandono del fruto de origen por parte de la hembra y termina con la respuesta 
a los estímulos del hospedero. Se ha sugerido que algunos Scolytinae vuelan aleatoriamente, sin ser atraídos hacia sus hospederos por ningún tipo de estímulo (Raffa y Berriman, 1983; Moeck et al., 1981; Byers, 1996). En términos evolutivos, se plantea que la etapa de vuelo y dispersión representa una alta vulnerabilidad para los individuos, que están expuestos a condiciones ambientales adversas, predadores y alto gasto energético, por lo que la búsqueda aleatoria de hospederos tendría un alto costo para la viabilidad de las especies. Por el contrario, los mecanismos que permiten reducir este periodo de exposición y con ello la mortalidad y el gasto energético, incrementan la viabilidad de la especie. Uno de tales mecanismos es la identificación del hospedero a través del reconocimiento de sus compuestos volátiles y la orientación del vuelo en función de dichos compuestos. Este tipo de atracción ha sido reportado ampliamente en especies de Scolytinae (Pearson, 1931; Bedard, 1966; Byers et al., 1979; Chapman, 1962; Cobb et al., 1968; Coster y Gara, 1968; Johnson y Coster, 1978; Moeck, 1970; Moeck, 1981; Renwick y Vité, 1969; Stark et al., 1968; Visser, 1986; Vité y Pitman, 1969; Wood, 1972). En la broca del café ha sido comprobada la atracción de las hembras en respuesta a alcoholes y mezclas de alcoholes (Benassi, 1990; Mendoza, 1991; Mathieu et al., 1997b, González y Dufour, 2000; Borbón et al., 2000; Cárdenas, 2000; Villacorta et al., 2001). Es oportuno recordar que en el caso de la broca del café, solamente las hembras migran y son responsables del establecimiento de la especie en nuevas áreas; los machos son ápteros y permanecen toda su vida dentro del fruto de origen.

El conjunto de características comportamentales que muestran las hembras con capacidad migratoria ha sido denominado comportamiento de abandono (Mathieu et al., 2001). Estas hembras han sido fecundadas antes de abandonar el fruto donde emergieron, poseen alas funcionales, y salen del fruto por efecto de las lluvias (Baker et al., 1984) o la alta insolación (Mathieu et al., 1997a). Durante el periodo de crecimiento del fruto, las hembras emergen de forma continua en busca de nuevos frutos. Al finalizar la cosecha, particularmente en Centroamérica, se dan migraciones masivas de la broca, que abandona los cultivos debido a la mínima oferta de frutos durante este periodo. Sin embargo, una parte de ellas permanece en los frutos sobremaduros que no fueron cosechados. En estos frutos la emergencia y la oviposición se detienen por completo, y se encuentra una gran concentración de adultos. Este fenómeno, conocido como "diapausa reproductiva" no ha sido estudiado a profundidad en el caso de la broca, pero se acentúa en las regiones donde el periodo entre cosechas es largo y se presenta marcada escasez de frutos durante el mismo (Mansingh, 1991). Las lluvias fuertes que inducen la nueva floración del cafeto promueven la ruptura de esta diapausa, con lo que se da la emergencia masiva de hembras de los frutos secos (Barrera et al., 2004b). El término "brocas en tránsito" se emplea para referirse a estos individuos que provienen de los frutos secos del año anterior, que tras el rompimiento de la diapausa reproductiva inician la búsqueda de nuevos frutos. Los individuos tienen una gran capacidad de vuelo, como se ha podido comprobar bajo condiciones de laboratorio, donde se han registrado tiempos de 90 min de vuelo continuo y 180 min de vuelos intermitentes (Bustillo y Villalba, 2004). Sin embargo, no todas las hembras migran, pues algunas nunca abandonan el fruto de origen, y solo aquellas que lo abandonan presentan una respuesta marcada a los estímulos olfativos. Aunque es una condición indispensable que la hembra haya sido fecundada para que abandone el fruto, hay una proporción de hembras que pese a haber sido fecundadas, no abandonan el fruto (Da Silva et al., 2006; Mathieu et al., 2001). Esto puede deberse a múltiples factores como la falta de desarrollo alar, carencia de depósitos de grasa, que constituyen la reserva de energía para el vuelo, o a una baja "propensión al vuelo", factores que a su vez pueden estar sujetos a control genético (Roff y Fairbairn, 2007). Es necesario realizar investigaciones que permitan una mejor comprensión de este fenómeno en la broca del café. Las hembras que no abandonan los frutos tampoco responden 
a los estímulos olfativos y su progenie se desarrolla en el mismo fruto de origen (Mathieu et al., 2001). En algunos Scolytinae ha sido reportada la necesidad de alguna cantidad de vuelo como requisito para la percepción de estímulos olfativos (Borden, 1967; Wood y Bushing, 1963). Se requieren trabajos de investigación que permitan una mejor comprensión de la relación entre capacidad de vuelo y capacidad de respuesta a estímulos olfativos en la broca.

\section{SELECCIÓN}

Comprende el periodo entre la percepción de los estímulos olfativos provenientes del hospedero hasta el inicio de la alimentación de la hembra sobre el sustrato (Wood, 1982). En cuanto al aterrizaje, el proceso podría ser aleatorio (Wood, 1982; Franklin et al., 2000; Franklin y Grégoire, 1999; Raffa y Berriman, 1983; Moeck et al., 1981; Byers, 1996; Gara et al., 1984), en el cual los individuos posarían indiscriminadamente sobre hospederos y no hospederos, sin embargo esto puede ser interpretado como una primera fase de aproximación a la zona apropiada y no necesariamente al fruto específico. Los modelos de selección de hospedero que involucran integración de estímulos visuales y químicos han adquirido relevancia en años recientes (Campbell y Borden, 2006a; Campbell y Borden, 2006b). Dicha integración también ha sido propuesta para la broca del café por Mathieu et al. (2001), quienes plantean que una vezla hembra entra en la etapa de selección, los estímulos visuales, constituidos por la percepción del color y, posiblemente, la forma y el tamaño, podrían integrarse con los estímulos olfativos, para permitir a la hembra su llegada a una baya en el estado de madurez óptimo y continuar con las etapas de concentración y establecimiento. También Bustillo y Villalba (2004) proponen que la broca es inicialmente atraída por el olor, y luego por el color y la forma del fruto. Este planteamiento concuerda con lo encontrado por Mendoza (1991), quien ha verificado la respuesta diferencial de las hembras tanto a estímulos ol- fativos de cuatro estados de desarrollo del fruto, como a sus respectivos colores.

\section{CONCENTRACIÓN}

En los Scolytinae de los cultivos forestales, esta etapa se da una vez que los primeros individuos colonizadores, previa evaluación de la aptitud del sustrato, comienzan a liberar feromonas de agregación que pueden tener un efecto sinérgico con volátiles de la planta, para orientar al resto de la población hacia su lugar de ubicación y así generar una concentración alta de la población en el hospedero (Wood, 1982). A este proceso se le ha denominado "atracción secundaria", en contraposición a la "atracción primaria", que se da cuando los primeros individuos colonizadores deben por sí mismos ubicar un sustrato adecuado para la alimentación y reproducción, únicamente mediante señales químicas del hospedero, o al azar. El tiempo transcurrido entre la detección del hospedero adecuado y la producción de feromona de agregación, es un aspecto importante, pues un lapso de tiempo pequeño implica una mayor sobrevivencia de la población de adultos buscando hospedero, que reducen su gasto energético y su exposición a predadores y condiciones ambientales adversas. En varios trabajos (Mendoza, 1991; Bustillo y Villalba, 2004; Bustillo, 2006) se plantea la posibilidad que en Hypothenemus hampei existan los dos tipos de atracción, pues ocurre una atracción primaria mediada por los estímulos químicos y visuales antes mencionados, y posteriormente podría darse una etapa de atracción secundaria, mediante sustancias producidas por las brocas colonizadoras, que podrían actuar en forma sinérgica con los volátiles del café. Mendoza (1991) reporta que tanto frutos verdes como maduros ejercen una mayor atracción sobre las hembras cuando ya han sido atacados, y la agregación de las poblaciones en campo reportada inicialmente por Baker (1984), es consistente con la presencia de una feromona que genere dicha agregación. Las evidencias indican que estas feromonas de 
agregación podrían encontrarse en las heces de las hembras (Bustillo y Villalba, 2004; Bustillo, 2006). Sin embargo hasta ahora no existen reportes de compuestos aislados de las heces, o de cualquier otra parte de $H$. hampei, como posibles feromonas de agregación, lo que a su vez impide estudiar aspectos más específicos como el tiempo transcurrido entre la llegada a los frutos y la producción de la feromona y la medida en que este factor puede reducir la mortalidad de hembras buscando nuevos granos. Esto pone de manifiesto la necesidad de realizar trabajos de investigación sobre los procesos involucrados en la etapa de concentración en $H$. hampei.

\section{ESTABLECIMIENTO}

Esta etapa inicia con la perforación de las galerías, previa evaluación de la calidad del recurso y concluye con la oviposición (Wood, 1982). En $H$. hampei se han reportado diferencias en el tiempo de perforación de los frutos, según su estado de desarrollo: se emplea un tiempo de perforación de $5 \mathrm{~h}$ y $36 \mathrm{~min}$ en los frutos verdes, 5 h y 54 min en frutos pintones, 4 h y 50 min en frutos maduros, y $11 \mathrm{~h}$ y $21 \mathrm{~min}$ para penetrar frutos sobremaduros (Bustillo y Villalba, 2004). Es evidente la penetración más rápida en los frutos maduros. En el desarrollo temprano de los frutos $(<120 \mathrm{~d})$ el porcentaje de materia seca inferior al $20 \%$ en los frutos, hace que su consistencia sea inapropiada para la perforación de la galería. Si esto ocurre, la hembra permanece en un pequeño canal de penetración cercano a la superficie, esperando a que el fruto alcance la consistencia adecuada. Durante este periodo el individuo es muy vulnerable pues se encuentra muy cerca del exterior del fruto. El inicio de la oviposición puede tardar desde 63 días después de la llegada de la hembra, si el fruto aún es demasiado verde (89\% de humedad) hasta cuatro días en frutos maduros (66\% de humedad). En la broca del café, al igual que en Trypodendron lineatum Oliv. (Coleoptera: Curculionidae: Scolytinae), las alas se degeneran después de que ha ocurrido perforación u oviposición (Mathieu et al., 2001). Debido a esto la hembra no abandona el fruto una vez que ha iniciado la perforación de la galería, y generalmente se presenta una sola hembra por fruto (Barrera, 2004; Borbón, 2004). De acuerdo con Cantor et al. (2000) esto podría deberse a algún mecanismo de marcación química, pues no se observan marcas físicas evidentes, o también a un posible comportamiento territorial, dado que estos autores reportan la defensa del grano por parte de la hembra residente cuando una segunda hembra intenta establecerse en el mismo fruto. Sin embargo, recientemente se ha observado en cultivos de la zona cafetera colombiana la presencia de perforaciones de varias hembras fundadoras en un mismo fruto, principalmente en las ramas del tercio inferior de la planta, donde el porcentaje de frutos con perforaciones múltiples oscila entre 2,0 y 16,5\% (Benavides et al., 1997).

Finalmente es importante resaltar que los mecanismos de atracción primaria y secundaria, mediante compuestos producidos por la planta y una posible feromona respectivamente, son temas de estudio promisorios con miras a la obtención de compuestos atrayentes más eficientes que permitan obtener niveles significativos de control de la plaga bajo las condiciones colombianas. 


\section{REFERENCIAS BIBLIOGRÁFICAS}

Baker, P.S. 1984. Some aspects of the behaviour of the coffee berry borer (Coleoptera:Scolytidae) in relation to its control in southern México. Folia Entomol. Mexic. 62, 9-24.

Barrera, J.F.; J. Herrera y J. Valle. 2005. Efecto de la altura de la trampa en la captura de la "broca del café": Implicaciones en dispersión y muestreo. Entomología Mexicana 4, 542-546.

Barrera, J.F.; A. Villacorta; J. Herrera; H. García y L. Cruz. 2004. Aplicación de trampas para el monitoreo de la broca del café. pp. 95-113. En: Néri A. (ed.). Manejo da broca do café. Workshop Internacional, Londrina, Brasil.

Bedard, W.D. 1966. Variation in capacity of Ips confusus to reach attractive hosts. pp. 137-142. En: Gerhold, H.D.; E.J. Schreiner; R.E. McDermott y J.A. Winieski (eds.). Breeding pest resistant trees. Pergamon, Oxford, U.K.

Benassi, V.L.R.M. 1990. Resultados preliminares da flutuação populacional da broca-do-café Hypothenemus hampei (Ferrari, 1867) (Coleoptera:Scolytidae) na região norte do Espírito Santo. p. 83. En: XVI Congressso Brasileiro de Pesquisas Cafeeiras. Rio de Janeiro, Brasil.

Benavides, P.; L.M. Constantino; L. Navarro y F.E. Acevedo. 1997. Herencia de caracteres genéticos y determinación de endogamia en la broca del café (Hypothenemus hampei). p. 46. Resúmenes XXXIV Congreso de la Sociedad Colombiana de Entomología (Socolen). Cartagena, Colombia.

Borbón, M.O.; O.M. Alfaro; A.M. Oelschlager y L.M. González. 2000. Proyecto de trampas, atrayentes y repelentes para el control de la broca del fruto del cafeto, Hypothenemus hampei L. (Coleoptera: Scolytidae). pp. 331-348. En: XIX Simposio Latinoamericano de Caficultura. San José.

Borden, J.H. 1967. Factors influencing the response of Ips confusus to male attractant. Can. Entomol. 99,1164-1193.

Bustillo, A.E. 2006. Una revisión sobre la broca, $H y$ pothenemus hampei (Coleoptera: Curculionidae: Scolytinae), en Colombia. Rev. Colomb. Entomol. 32(2), 101-116.

Bustillo, A.E. y D.A. Villalba. 2004. Efecto del clima y condiciones de cultivo del café en la biología y comportamiento de la broca del café, Hypothenemus hampei (Ferrari) (Coleoptera: Curculionidae: Scolytinae). pp. En: Manejo da broca do café. Workshop Internacional. Londrina, Brasil.

Byers, J.A. 1996. An encounter rate model of bark beetle populations searching at random for susceptible host trees. J. Ecol. Modelling 91, 57-66.

Byers, J.A.; D.L. Wood; L.E. Browne; R.H. Fish; B. Piatek y L.B. Hendry. 1979. Relationship between a host plant compound, myrcene, and pheromone production in the bark beetle Ips paraconfusus. J. Insect Physiol. 25,477-482.

Campbell, S.A. y J. Borden. 2006a. Close-range, in flight integration of olfactory and visual information by a host-seeking bark beetle. Entomol. Exp. Appl. 120,91-98.

Campbell, S.A. y J. Borden. 2006b. Integration of visual and olfactory cues of hosts and non-hosts by three bark beetles. (Coleoptera: Scolytidae). Ecol. Entomol. 31, 437-449.

Cantor, F.; O. De Souza; E.F. Vilela; C.A. Faria y F Gumier-Costa. 2000. Local deterrence of multiple attacks by the coffee Berry borer. p. 164. En: Libro de resúmenes I: XXI International Congress of Entomology. Foz do Iguaçu, Brasil.

Cárdenas, R.M. 2000. Trampas y atrayentes para monitoreo de poblaciones de broca del café Hypothenemus hampei (Ferrari) (Col., Scolytidae). pp. 369379. En: Memorias XIX Simposio Latinoamericano de Caficultura. San José.

Cardona, G.E. y A.E. Bustillo. 2006. Captura de broca del café Hypothenemus hampei (Ferrari) usando trampas en tres densidades diferentes. p. 133. En: Resúmenes XXXIII Congreso de la Sociedad Colombiana de Entomología (Socolen). Manizales, Colombia.

Cardona, G.E. y A.E. Bustillo. 2007. Evaluación de trampas cebadas con alcoholes para la captura de la broca del café, Hypothenemus hampei (Ferrari) (Coleoptera: Scolytidae). p. 75. Resúmenes XXXIV Congreso de la Sociedad Colombiana de Entomología (Socolen). Cartagena, Colombia.

Chapman, J.A. 1962. Field studies on attack flight and log selection by the ambrosia beetle Trypodendron lineatum (Olivier). Can. Entomol. 94, 74-92. 
Cobb, F.W.; D.L. Wood; R.W. Stark y P.R. Miller. 1968. Photochemical oxidant injury and bark beetle infestation of ponderosa pine II. Effect of injury upon physical properties of oleoresin, moisture content and phloem thickness. Hilgardia 59, 127-134.

Coster, J.E. y R.I. Gara. 1968. Studies on the attack behaviour of the southern pine beetle II. Response to attractive material. Contrib. Boyce Thompson Inst. 24, 69-76.

Da Silva, F.C.; M.U. Ventura y L. Morales. 2006. Capture of Hypothenemus hampei Ferrari (Coleoptera: Scolytidae) in response to trap characteristics. Scientia Agric. 63(6), 567-571.

Dufour, B.P. y B. Frérot. 2008. Optimization of coffe Berry borer, Hypothenemus hampei Ferrari (Col., Scolytidae) mass trapping with an attractant mixture. J. Appl. Entomol. 132, 591-600.

Fernández, S. y Cordero J. 2005. Evaluación de atrayentes alcohólicos en trampas artesanales para el monitoreo y control de la broca del café, Hypothenemus hampei (Ferrari). Bioagro 17(3), 143-148.

Franklin, A.J.; C. Debruyne. y J.C. Grégoire. 2000. Recapture of Ips typographus L. (Col.,Scolytidae) with attractants of low release rates: localized dispersión and environmental influences. Agr. Forest Entomol. 2, 259-270.

Franklin, A. y J.C. Grégoire. 1999. Flight behaviour of Ips tipographus L. (Col., Scolytidae) in an environment without pheromones. Ann. Forest Sci. 56(7), 591-598.

Gara, R.I.; D.R. Geizler y W.R. Littke. 1984. Primary attraction of the mountain pine beetle Dendroctonus ponderosae to lodgepole pine, Pinus contorta in $\mathrm{Or}$ egon. Ann. Entomol. Soc. Amer. 77, 333-334.

González, M.O. y B.P. Dufour. 2000. Diseño, desarrollo y evaluación del trampeo en el manejo integrado de la broca del café Hypothenemus hampei. pp. 381-396. En: XIX Simposio Latinoamericano de Caficultura. Costa Rica.

Johnson, P.C. y J.E. Coster. 1978. Probability of attack by the southern pine beetle in relation to distance from an attractive host tree. Forestry Sci. 24, 574580

Lindgren, B.S. 1983. A multifunnel trap for scolytid beteles (Coleoptera). Can. Entomol. 115(3), 299. 302

Mansingh, A. 1991. Limitations of insecticides in the management of the coffe berry borer Hypothenemus hampei Ferrari. J. Coffee Res.
Mathieu, F; L.O. Brun y B. Frérot. 1997a. Factors related with native host abandon by the coffee berry borer. J. Appl. Entomol. 121, 181-186

Mathieu, F.; L.O. Brun; C. Marchillaud y B. Frérot. 1997 b. Trapping of the coffee berry borer Hypothenemus hampei Ferr. (Col., Scolytidae) within a mesh-enclosed environment: Interaction of olfactory and visual stimuli. J. Appl. Entomol. 121(3), 181-186.

Mathieu, F. ; V. Gaudichon; L.O. Brun y B. Frérot. 2001. Effect of physiological status on olfactory and visual responses of female Hypothenemus hampei during host plant colonization. Physiol. Entomol. 26, 189-193.

Mendoza, M.J.R. 1991. Resposta da broca-do-café, Hypothenemus hampei, a estímulos visuais e semioquímicos. Dissertaçao de Mestrado. Universidad Federal de Viçosa, Brasil.

Moeck, H.A. 1970. Ethanol as the primary attrctant for the ambrosia beetle Trypodendrum lineatum (Coleoptera: Scolytidae). Can. Entomol. 102, 792-796.

Moeck, H.A. 1981. Ethanol induces attack on tres by spruce beteles, Dendroctonus rufipennis (Coleoptera: Scolytidae). Can. Entomol. 113(10), 939-942.

Ortíz, A.; F.E. Vega y F.J. Posada. 2004. Volatile composition of coffee berries at different stages of ripennes, and their possible attraction to the coffee berry borer Hypothenemus hampei (Coleoptera: Curculionidae). J. Agr. Food Chem. 52, 5914-5918.

Pearson, H.L. 1931. Theory in explanation of the selection of certain trees by the western pine beetle. J. Forestry 29, 696-699.

Prates, H.S. 1969. Observações preliminares de atração da broca-do-café Hypothenemus hampei (Ferrari, 1867) a extratos de frutos de cafeeiro (cereja e verde). O Solo 61(2), 13-14

Raffa, K.F. y A.A. Berryman. 1983. The role of host plant resistance in the colonization behaviour and ecology of bark beetles (Coleoptera: Scolytidae). Ecol. Monogr. 53, 27-49.

Renwick, J.A. y J.P. Vité. 1969. Bark Beetle Attractants: Mechanisms of colonization by Dendroctonus frontalis. Nature 224, 1222-1223.

Roff, D.A. y D.J. Fairbairn. 2007. The evolution and genetics of migration in insects. BioScience $57(2)$, 155-164.

Stark, R.W.; P.R. Miller; F.W. Cobb; D.L. Wood y J.R. Parmeter. 1968. Photochemical oxidant injury and bark beetle infestation of ponderosa pine I. Inci- 
dence of bark beetle infestation of ponderosa pine. Hilgardia 39, 121-126.

Ticheler, J. H. G. 1963. Estudio analítico de la epidemiología del escolitídeo de los granos del café Stephanoderes hampei Ferr. en Costa de Marfil. Revista Cenicafé 14(4), 223-294.

Villacorta, A.; A.F. Possagnolo; R.Z. Silva y P.S. Rodrigues. 2001. Um modelo de armadilha com semioquímicos para o manejo integrado da broca do café Hypothenemus hampei (Ferrari) no Paraná. pp. 2093 2098. En: Simpósio de Pesquisa dos Cafés do Brasil. Vitória, Brasil.

Visser, J.H. 1986. Host odour perception in phytophagous insects. Annu. Rev. Entomol. 31, 121-144
Vité, J.P. y G.B. Pitman. 1969. Insect and host odours in the aggregation of the western pine beetle. Can. Entomol. 101, 113-117.

Wood, D.L. 1972. Selection and colonization of ponderosa pine by bark beetles. pp. 101-117. En: Van Emden, H.F. (ed.). Plant relationships. Blackwell Science, Oxford, UK.

Wood, D.L. 1982. The role of pheromones, kairomones and allomones in the host selection and colonization behaviour of bark beetles. Annu. Rev. Entomol. 27, 411-446.

Wood, D.L. y R.W. Bushing. 1963. The olfactory response of Ips confusus (Lec.) to the secondary attraction in the laboratory. Can. Entomol. 95, 1066-1078. 